\title{
Polycystic liver
}

disease - a disease entity presenting as part of autosomaldominant polycystic kidney disease

\section{H Boonzaier-Botha MB ChB \\ Department of Diagnostic Radiology University of the Free State and Universitas Hospital Bloemfontein \\ C Cock \\ MB ChB, MMed (Int) \\ Department of Gastroenterology University of the Free State and Universitas Hospital Bloemfontein}

\section{Abstract}

A 65-year-old man was referred to the Gastroenterology Department with complaints of longstanding upper abdominal discomfort and hepatomegaly. Ultrasound of the liver revealed a massively enlarged liver with multiple cystic areas. Aspiration of the largest cyst revealed $15 \mathrm{ml}$ of yellow fluid without any organisms or malignant cells. A diagnosis of autosomal-dominant polycystic kidney disease was suspected, but could not initially be confirmed on ultrasound/ computed tomography imaging. Magnetic resonance imaging confirmed the multiple hepatic cysts and revealed multiple smaller cysts in both kidneys. Symptomatology subsided after aspiration of the largest cyst and the patient was discharged. The patient has subsequently been followed up and is currently symptom free. The case illustrates the importance of screening for associated kidney disease in patients with polycystic liver disease.

\section{Introduction}

Polycystic liver disease (PCLD) occurs most commonly as a manifestation of all forms of polycystic kidney disease, but especially autosomaldominant polycystic kidney disease (ADPKD)..$^{1-5}$ It also occurs much more rarely as a disease entity on its own and is termed autosomaldominant polycystic liver disease (ADPLD). ${ }^{4-6}$ The association of multiple hepatic cysts with kidney disease and the clinical implications thereof are, however, of primary relevance, as is demonstrated by our clinical case.

Radiologically, PCLD must be differentiated from common hepatic cysts that occur in at least $2.5 \%$ of the population, multiple echinococcus cysts, cystic metastases, acquired cysts from trauma or infection, biliary cystadenoma and the clinically insignificant and rare Von Meyenburg complexes. ${ }^{78}$

\section{Case report}

A 65-year-old man was referred from a general practitioner to the Gastroenterology Department with vague complaints of longstanding upper abdominal discomfort and hepatomegaly. Past history was unremarkable other than the intake of traditionally brewed beer. Clinical examination revealed $21 \mathrm{~cm}$ hepatomegaly and the initial suspicion was that of alcoholic liver cirrhosis or haemosiderosis. Biochemical investigations done before the ultrasound appointment were aimed at excluding infections, neoplasms and impaired liver and kidney function. Liver enzymes and liver function tests were all normal other than a raised gammaglutamyl transpeptidase of $176 \mathrm{IU} / \mathrm{l}$ and a serum albumin slightly below normal at $35 \mathrm{~g} / \mathrm{dl}$. Uric acid was increased to $0.48 \mathrm{mmol} / \mathrm{l}$. Protein electrophoresis was normal, as were tests for haemostasis. The erythrocyte sedimentation rate was $31 \mathrm{~mm} / \mathrm{h}$ and C-reactive protein below $5 \mathrm{mg} / \mathrm{l}$. Virology for hepatitis A, B and C were all negative and serology was negative for echinococcus and bilharzia. Tumour markers including carcinoembryonic antigen, alpha-fetoprotein and prostate- specific antigen were in the normal reference range.

Ultrasound (Fig. 1) of the liver revealed a massively enlarged liver $10 \mathrm{~cm}$ below the ribs in the midaxillary line distending across the midline to the left hypochondrium. Multiple cystic areas with posterior enhancement were visible without any obvious characteristics of echinococcus 
cysts. A single simple cortical cyst measured $21 \mathrm{~mm} \times 20 \mathrm{~mm}$ in the lower pole of the left kidney.

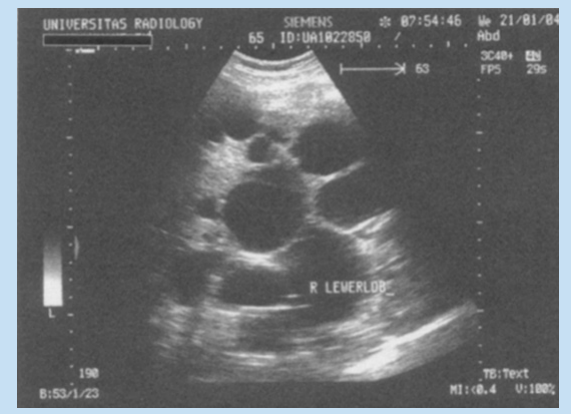

Fig.1. Ultrasound of enlarged liver.

Helical computed tomography (CT) scan (GE Prospeed SX Advantage Helical) pre IV contrast (Fig. 2a) and post IV contrast (Fig. 2b) with oral contrast confirmed the multiple hepatic cysts and single simple cortical cyst in the lower pole of the left kidney. The multiple liver cysts varied in size with a maximum diameter of approximately $8 \mathrm{~cm}$ and no enhancement post intravenous contrast injection. A single enlarged pre-aortic node was visible without notable abnormalities in the rest of the abdominal structures. Aspiration of the largest cyst revealed $15 \mathrm{ml}$ of yellow fluid without any organisms or malignant cells.

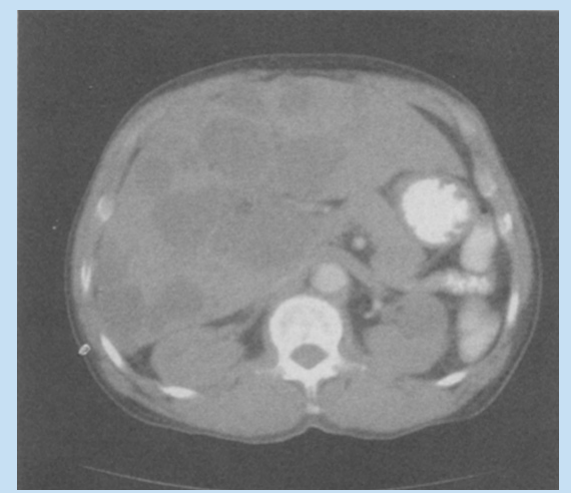

Fig. 2a. Helical computerised tomography pre IV contrast confirming hepatic and cortical cysts.

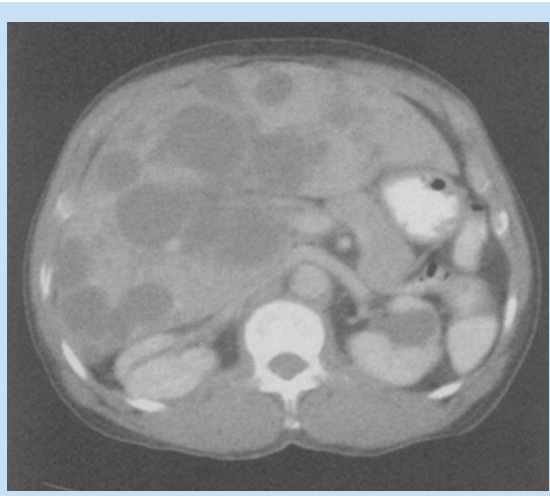

Fig. 2b. Helical computerised tomography post IV contrast confirming hepatic and cortical cysts.

Magnetic resonance imaging (MRI, GE Signa Twinspeed $1.5 \mathrm{~T}$ unit) confirmed the multiple hepatic cysts with low signal on T1-weighted images (Fig. 3a) and high signal on T2-weighted images (Figs $3 \mathrm{~b}$ and 3c) without enhancement post gadolinium injection. The hepatic cysts were initially accurately imaged with ultrasound and no extra information regarding the characteristics of the hepatic cysts was revealed with CT or MR investigations. These investigations were done for comparative purposes and confirmation of initial diagnosis of PCLD. MR imaging, however, revealed multiple smaller cysts in both kidneys (Fig. 3d). A diagnosis of ADPKD, which had not been suspected on clinical features or ultrasound/CT imaging, was made.

Based on the MRI findings a 24hour urine protein concentration and creatinine clearance were done, but were found to be within normal limits. The patient was not hypertensive and fundoscopic examination was normal.

Symptomatology subsided after aspiration of the largest cyst and the patient was discharged with the addition of a short course of sucralfate. He has subsequently been followed up at the Gastroenterology Clinic and is

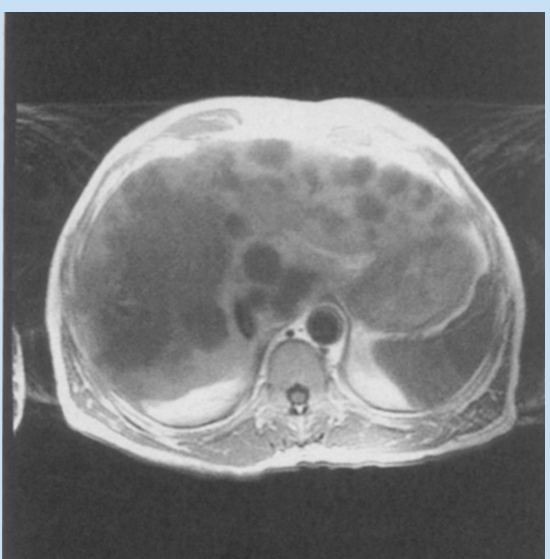

Fig. 3a. MRI confirming multiple hepatic cysts with low signal on T1-weighted images.

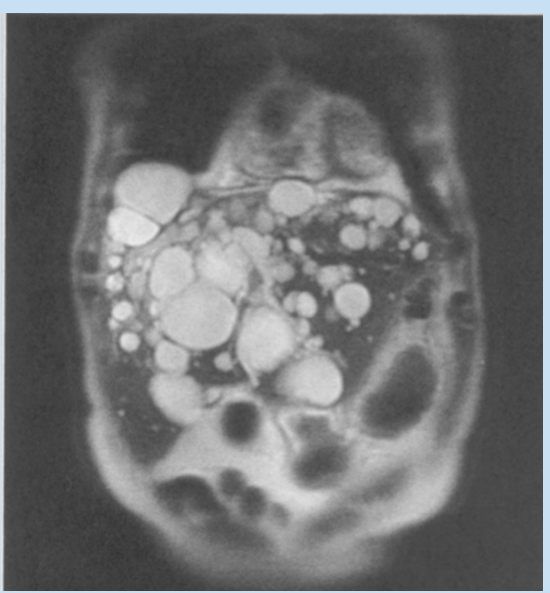

Fig. 3b. MRI confirming multiple hepatic cysts with high signal on T2-weighted images.

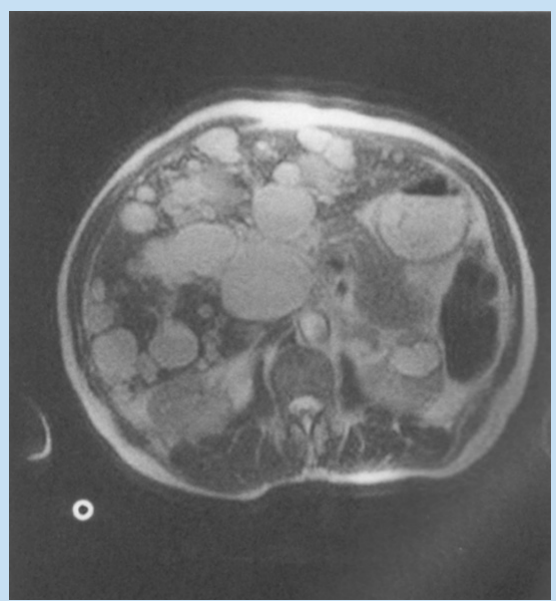

Fig. 3c. MRI confirming multiple hepatic cysts with high signal on T2-weighted images. 


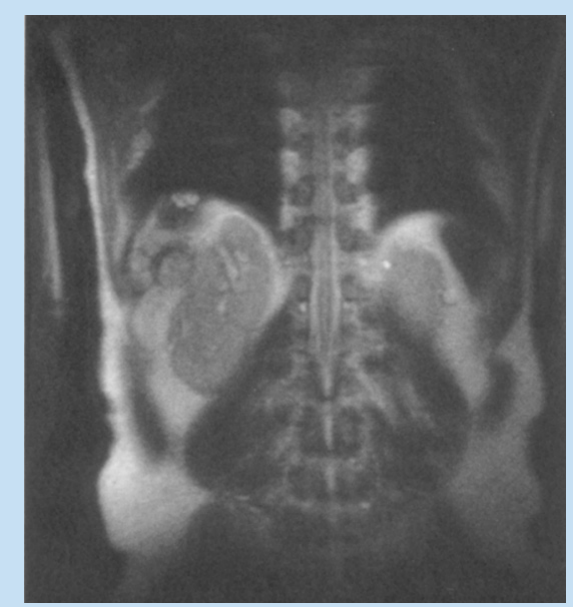

Fig. 3d. MRI confirming multiple smaller cysts in both kidneys.

currently symptom free. Screening of his living relatives, by ultrasound and biochemistry, has revealed active ADPKD in one of his three children.

\section{Discussion}

PCLD usually occurs as a manifestation of ADPKD and can occur with ADPKD-1 (type 1) and ADPKD-2 (type 2) ${ }^{1-5}$ It can, however, also rarely occur as a separate entity distinct from $\mathrm{ADPKD}^{4-6}$ or a manifestation of autosomal recessive polycystic kidney disease. $^{2,9,10}$

In adult patients PCLD is most commonly associated with ADPKD types 1 and 2. In these disorders there is abnormal formation of the protein polycystin, coded for by chromosome 16 (ADPKD type 1 ) and chromosome 4 (ADPKD type 2). ${ }^{3.5}$ In primary PCLD there is abnormal formation of hepatocystin coded for by chromosome19. ${ }^{11-13}$ Polycystin and hepatocystin play a role in the processing of secretary and transmembrane proteins that include receptors for the control of cell proliferation in the liver, kidneys, pancreas and spleen (and abnormalities thereof may lead to cyst formation in these organs)., ${ }^{4,1,15}$
In children PCLD may be associated with congenital hepatic fibrosis and autosomal recessive polycystic kidney disease. The disease process in the recessive form is much more malignant and survival into adulthood is rare. ${ }^{4}$

Radiologically the disease is characterised by the presence of multiple (4 or more) cysts imaged on ultrasound, CT or MRI. ${ }^{7}$ Hepatic cysts are presumed to occur in $2.5 \%$ of the population and can be single or multiple as part of ADPKD-1 and 2. They are developmental anomalies originating from hamartomatous tissue. The cysts contain serous fluid and are lined by a thin wall of cuboidal epithelium, identical to that of the bile ducts, with a fibrous stroma underneath.? Most cysts are asymptomatic but onesixth of them cause symptoms due to liver enlargement or pressure on surrounding organs. ${ }^{16}$

Malignant degeneration of hepatic cysts is very rare but has to be excluded by means of aspiration cytology before diagnosis is confirmed or symptomatic cysts are treated. Simple cysts are round, oval lesions with posterior enhancement and are echo-free. Thin septations can usually be seen on imaging. ${ }^{8}$ Associated cysts can be found in the kidneys in $50 \%$ of cases as well as in the pancreas, spleen and other organs. ${ }^{8}$ Ultrasound is usually superior to CT or MRI and depicts the well-defined, anechoic, round or oval lesions with imperceptible walls, thin septae and good posterior enhancement or through transmission.

Ultrasound is also used for aspiration of cystic contents and interventional treatment of symptomatic cysts. The cysts can be inhomogeneous due to haemorrhage inside and must then be differentiated from cys- tic metastasis. ${ }^{7}$ Haemorrhage and infection can cause debris-like echoes in the affected cysts on ultrasound which are then difficult to differentiate from cystic metastases that occur for example in squamous cell carcinoma and gastric stromal tumours. ${ }^{8}$ Hydatid disease of the liver can usually be differentiated by the typical sonographic appearance of the cyst wall and due to visible bands of delaminated endocysts. This is usually described as a 'water-lily sign'. Calcification of the echinococcus cyst wall and daughter cysts that are surrounded by an echogenic debris, sometimes called hydatid sand, is frequently seen. On non-enhanced CT scans the multiple cysts appear mostly homogeneous and hypodense with no enhancement of the wall or cyst contents after contrast administration. ${ }^{7,17}$

On MRI the hepatic cysts are characteristically of low image signal intensity on $\mathrm{T} 1$ and have very high signal intensity on T2-weighted images. No enhancement is seen of wall or cyst contents after injection of gadolinium chelates. Once again slight variations can occur in signal intensity due to intracystic haemorrhage or infection.

PCLD is most commonly a clinically benign condition and in most cases presents as vague upper abdominal discomfort or abnormal liver enzyme tests detected incidentally. There is often a positive family history or previous diagnosis of polycystic kidneys. In a large number of cases liver function tests remain normal. The disease usually occurs in older patients who often present with nodular liver enlargement and a normal liver function test. When PCLD occurs in association with ADPKD the renal function may become 
abnormal.

In rare cases cysts may become massively enlarged or may become infected. Even rarer complications are Budd-Chiari syndrome, portal hypertension, or liver failure but these may be so severe that liver transplantation may be indicated. ${ }^{4.18}$

Treatment initially consists of treating symptomatic cysts, which are usually the largest cysts imaged, with unroofing, excision or hepatic resection. ${ }^{16}$ In PCLD it is, however, advisable to treat the symptomatic cyst with injection of $25-30 \%$ of the aspirated cyst volume using $99 \%$ ethanol, with re-aspiration after 20 minutes. ${ }^{16}$ Sonography is usually the most accurate imaging method for evaluation and treatment of symptomatic cysts under ultrasound-guided aspiration and ethanol injection. ${ }^{16}$ Arterial embolisation of liver disease represents a new and exciting development in the treatment of patients in poor physical condition. In one case symptomatic relief was achieved as well as a reduction in hepatomegaly and ascites with improvement in physical condition due to improved appetite. ${ }^{19}$

In summary, PCLD occurs as part of the manifestation of ADPKD in more than $50 \%$ of cases. Where the association occurs it is clinically relevant as the patient will much more likely suffer renal complications, with the liver disease running a relatively benign course. Radiologically, it must be distinguished from other cystic diseases of the liver. The most common of these are multiple metastases, hydatid disease, acquired cysts from trauma or infection and biliary cystadenoma.

Radiological investigations play a crucial role in suggesting and confirming the correct diagnosis and managing patients appropriately.

\section{References}

1. Gabow PA. Autosomal dominant polycystic kidney disease. $N$ Engl J Med 1993; 329: 332 342

2. D'Agata ID, Jonas MM, Perez-Atayde AR, Guay-Woodford LM. Combined cystic disease of the liver and kidney. Semin Liver Dis 1994; 14 215-228.

3. Rizk D, Chapman AB. Cystic and inherited kidney diseases. Am J Kidney Dis 2003; 42: 13051317.

4. Sherlock SA, Dooley J. Diseases of the Liver and Biliary System. 11th ed. Oxford: Blackwell Science, 2002: 584-586

5. Feldman M, Friedman LS, Sleisenger MH Sleisenger \& Fordtran's Gastrointestinal and Liver Disease. 7th ed. Philadelphia: WB Saunders, 2002: 1596-1597.

6. Karhunen PJ, Tenhu M. Adult polycystic liver and kidney diseases are separate entities. Clin Genet 1986; 30(1): 29-37.

7. Mortele KJ, Ros PR. Imaging of diffuse liver disease. Semin Liver Dis 2001; 21: 195-212.

8. Ralls PW, Jeffrey RB Jr, Kane RA, Robbin M. Ultrasonography. Gastroenterol Clin North Am
$2002 ; 31: 801-825$

9. Lai EC, Wong J. Symptomatic nonparasitic cyst of the liver. World J Surg 1990; 14: 452-456.

10. Summerfield JA, Nagafuchi Y, Sherlock S, Cadafalch J, Scheuer PJ. Hepatobiliary fibropolycystic diseases. A clinical and histological review of 51 patients. J Hepatol 1986; 2: 141 156.

11. Drenth JP, te Morsche RH, Smink R, Bonifacino IS, Jansen JB. Germline mutations in PRKCSH are associated with autosomal dominant polycystic liver disease. Nat Genet 2003; 33: 345-347.

12. Li A, Davila S, Furu L, et al. Mutations in PRKC$\mathrm{SH}$ cause isolated autosomal dominant polycystic liver disease. Am J Hum Genet 2003; 72: 691-703.

13. Minoshima $S$, Hirai $M$, Sakai $K$, et al. Assignment of the gene for a protein kinase $C$ substrate, $80 \mathrm{~K}$ protein, to human chromosome 19 (Abstract). Cytogenet Cell Genet 1989; 51: 1045.

14. Hateboer N, v Dijk MA, Bogdanova N, et al Comparison of phenotypes of polycystic kidney disease types 1 and 2. European PKD1 PKD2 Study Group. Lancet 1999; 353: 103-107.

15. Ibraghimov-Beskrovnaya O, Dackowski WR Foggensteiner L, et al. Polycystin: in vitro synthesis, in vivo tissue expression, and subcellula localization identifies a large membrane-associated protein. Proc Natl Acad Sci USA 1997; 94: 6397-6402.

16. Tikkakoski T, Makela JT, Leinonen S, et al. Treatment of symptomatic congenital hepatic cysts with single-session percutaneous drainage and ethanol sclerosis: technique and outcome. J Vasc Interv Radiol 1996; 7: 235-239.

17. Murphy BJ, Casillas J, Ros PR, Morillo G Albores-Saavedra J, Rolfes DB. The CT appearance of cystic masses of the liver. Radiographics 1989; 9: 307-322.

18. Uddin W, Ramage JK, Portmann B, et al Hepatic venous outflow obstruction in patient with polycystic liver disease: pathogenesis and treatment. Gut 1995; 36: 142-145.

19. Ubara Y, Takei R, Hoshino J, et al. Intravascular embolization therapy in a patient with an enlarged polycystic liver. Am J Kidney Dis 2004 43: 733-738. 